pansion of such a universe give the observed spectral shifts, and could the rate of replacement make up for the great rate of loss calculated by Mr. Hoyle? To answer these questions is beyond my mathematics, and until the answers are forthcoming my theory had better be shelved.

But I am not content with Mr. Hoyle's theory either. According to this the size of a nebula is proportional to its age. Although it is not probable that even one very old and large one would occur within our own optical horizon, we should expect to observe a complete range of sizes, from quite new and small ones up to a limit of size set by probability considerations. Do we? Until it has been proved that the size distribution of observable nebulæ is what should be expected on Mr. Hoyle's theory, I suggest, reluctantly, that one cannot be wholly convinced of the soundness of this attractive theory according to which matter keeps on drifting out of sight.

About the size distribution of nebulæ I would suggest that my theory seems to have the advantage, for I think it is consistent with an upper limit to the possible concentration of matter in any one place and also with a rare occurrence of small concentrations. According to my theory, small incipient accumulations of matter would drift together under their mutual gravitational attraction. (According to Mr. Hoyle, as I understand him, they would separate by expansion of the universe too quickly to do this.) The groups of accumulations that I am assuming would grow both by attracting new diffuse matter from the environment and by drifting together with other groups to form groups of groups. But I think it could be shown that such a process of concentration cannot go on indefinitely.

Let the total matter in a fair sample of the universe be $\alpha M$ in the concentrations (nebulæ) and $(1-\alpha) M$ in interstellar space. The loss-rates will then be $\alpha M / L$ in the nebulæ and $(1-\alpha) M / L$ in interstellar space. Replacement-rate of the nebulæ from diffuse matter will depend on the average time $S$ taken by a particle during its fall as well as on the proportion $\beta$ of particles that complete the journey before disappearing, $\beta$ being, of course, a function of $L$. The net rate of gain of the nebulæ in a fair sample will therefore be :

$$
G=\beta(1-\alpha) M / S-\alpha M / L .
$$

As accumulations of matter drift together into greater concentrations the loss of matter will become more localized, while the appearance of new matter will continue to be evenly distributed in interstellar space. So the nebulæ will have to draw on greater distances for their compensations, and the proportion $\beta$ of particles that finish the longer journey will decrease. At the same time, the more intense gravita. tional field of the larger concentrations will lead to more rapid depletion of interstellar space and to an increase in $x$. One would, therefore, expect to find a limiting distance between nebulæ beyond which $G$ is negative and the nebulæ would dwindle. This limiting distance would limit also the process of grouping into larger and larger concentrations. One would also expect that small accumulations would grow rapidly, so that not many of them would be observable at any given moment.

The above considerations lead me to hope that Mr. Hoyle, and others with more mathematical skill than I possess, may give their further attention to both theories about the manner in which matter disappears. I should not bo surprised if, as a result, a third and better theory of disappearance were to emerge.

$$
\begin{aligned}
& \text { University College, } \\
& \text { London, W.C.1. } \\
& \text { Jan. 20. } \\
& \text { Large-Area Scintillation Counters }
\end{aligned}
$$

\section{Large-Area Scintillation Counters}

IT is of great interest, particularly in cosmic ray experiments, to employ scintillation counters which have a large sensitive area. The first essential is a large transparent crystal. Crystals of naphthalene containing 1 per cent of anthracene and of size up to $8 \mathrm{in}$. in diameter and $\frac{1}{2}$ in. thick have been grown, and undoubtedly it will be possible to use large crystals of other substances in the future. Here, however, we are concerned only with the optical problems which arise when large crystals are used. The aim of this note is to direct attention to a useful development in this field.

Some optical device is required to ensure that the light produced in different regions of the crystal is collected by the phototube in a uniform and efficient way. A cone, paraboloid, hemisphere and a sphere were compared by scanning the plane, which would normally contain the large crystal, with a small anthracene crystal behind which was fixed a polonium $\alpha$-source. We found that the best results were obtained with an integrating sphere having a highly reflecting diffuse white surface of magnesium oxide.

The photosensitive surface of the phototube projects into such a sphere, and the large crystal (of diameter slightly less than that of the sphere) is supported in a diametral plane facing the photosensitive surface. The signal given by a scintillation produced at the edge of the crystal is only 10 per cent less than that produced by one at the centre. Very little loss is sustained if a hemisphere or a paraboloid is used with the crystal backed by a flat plate coated in the same manner. In general, a diffuse reflecting system of this type was found to be nearly twice as efficient as a specular reflecting system of the same dimensions. With the latter, the light collected from each scintillation by the phototube is limited to several distinct cones, whereas, with diffuse reflexion, light from any scintillation can reach the phototube.

This method of diffuse reflexion in large-area scintillation counters is now being used in cosmic-ray research at this Establishment.

$$
\begin{aligned}
& \text { R. BatCHELoR } \\
& \text { J. W. SMITH } \\
& \text { L. BLACKMORE* } \\
& \text { Atomic Energy Research Establishment, } \\
& \text { Harwell, Nr. Didcot, Berks. } \\
& \text { Jan. 23. }
\end{aligned}
$$

* Of the Royal Naval Scientific Service, Teddington, Middlesex.

\section{Excess of Positive Mesons in Cosmic Rays}

The excess of positive mesons in cosmic rays at sea-level led to the original theory of the production of mesons by primary protons. It seems probable that precise measurements of the ratio of positive to negative mesons will help to decide between the several processes suggested in the literature.

This communication reports preliminary measurements of the meson momentum spectrum made with 